\title{
HIF1 $\alpha$-associated circDENND4C Promotes Proliferation of Breast Cancer Cells in Hypoxic Environment
}

\author{
GEHAO LIANG ${ }^{1 *}$, ZIHAO LIU $^{1 *}$, LUYUAN TAN $^{1}$, AN SU $^{1}$, WEN G. JIANG ${ }^{2}$ and CHANG GONG ${ }^{1,2}$ \\ ${ }^{1}$ Guangdong Provincial Key Laboratory of Malignant Tumor Epigenetic and Gene Regulation, \\ Breast Tumor Center, Sun Yat-sen Memorial Hospital, Sun Yat-sen University, Guangzhou, P.R. China; \\ ${ }^{2}$ Cardiff China Medical Research Collaborative, Cardiff University School of Medicine, \\ Cardiff University, Cardiff, U.K.
}

\begin{abstract}
Background: Accumulating evidence has shown that hypoxia plays a key role in regulating proliferation of breast cancer cells. However, the mechanism of how hypoxia regulates breast cancer remains unclear. We sought to investigate if hypoxia regulated proliferation through circular RNA. Materials and Methods: Western blot was used to detect hypoxia-inducible factor 1 alpha (HIFl $\alpha)$ levels in breast cancer cells under hypoxic conditions. Candidate circular RNAs (circRNAs) were selected and quantified by quantitative real-time polymerase chain reaction (qRT-PCR) after hypoxia induction. CCK8 assay was used to investigate the changes of proliferation after interfering circDENND4C and HIFla. Results: In breast cancer cells, circDENND4C was increased under hypoxic conditions and decreased after knocking-down HIFla. In addition, knocking-down circDENND4C inhibited proliferation of breast cancer cells in a hypoxic environment. Finally, tumors with a large size had higher circDENND4C expression levels than those of small size. Conclusion: CircDENND4C is a HIF1 $\alpha$-associated circRNA promoting the proliferation of breast cancer cells under hypoxia.
\end{abstract}

Hypoxia is a key feature of most solid tumors and affects a variety of tumor cell properties, such as proliferation, angiogenesis, metastasis, metabolism and autophagy (1-5). To adapt to hypoxia stress, cancer cells respond by increasing the expression of hypoxia-inducible factor 1 alpha (HIF1 $\alpha$ ).

*These Authors contributed equally to this work.

Correspondence to: Chang Gong, Sun Yat-sen Memorial Hospital, Sun Yat-sen University, 107 Yanjiang West Road, Guangzhou 510120, P.R. China. Tel: +86 13925089353, Fax: +86 2081332853, e-mail: changgong282@163.com

Key Words: HIF1 $\alpha$, circDENND4C, proliferation, hypoxia, breast cancer.
HIF1 $\alpha$, which works as a transcription factor, extensively regulates transcriptions of coding genes, such as $V E G F, I L-6$, and non-coding genes, such as lncRNAs and miRNAs (6-8), that promote angiogenesis, proliferation and metastasis of cancer cells. Hypoxia level is reported to be positively correlated to prognosis of patients with cancer (9). However, the role of HIF1 $\alpha$ in regulating some novel molecules, such as circular RNA (circRNA), remains unknown.

CircRNA is a novel type of RNA that forms a covalently closed continuous loop. CircRNAs were typically considered to be molecular flukes or byproducts of transcription. However, in recent years, a number of studies have indicated that circRNAs exhibited powerful functional potential in regulating proliferation and metastasis (10-12), suggesting that circRNAs may be important regulatory molecules in cancer. CircRNAs are very interesting in that, due to lack of 5' cap and 3' ends, they are not easily degraded by RNase R and are more stable than parent linear RNAs (13). Compared to HIF1 $\alpha$, HIF1 $\alpha$-associated circRNAs are, thus, more stable. In addition, circRNAs are abundant and conserved in mammalian cells, indicating circRNAs are ideal biomarker candidates for disease, including cancer, and several articles have identified certain circRNAs as biomarkers for predicting the prognosis of cancers, including esophageal, gastric and colon cancers (14-16). Therefore, identifying HIF1 $\alpha$-associated circRNAs with functional roles in cancer will have important clinical significance in translational medicine for biomarker-based clinical trials of cancer.

In the present study, we identified the existence of a hypoxia-associated circRNA, circDENND4C, in breast cancer cells and found that its expression was increased after hypoxia induction and decreased after knocking-down HIF l $\alpha$. Knocking-down circDENND4C inhibited proliferation of breast cancer cells in a hypoxic environment. In addition, the clinical relevance between circDENND4C expression and clinicopathological features were also analyzed in human breast cancer samples. Our findings indicated that circDENND4C is an HIF1 $\alpha$-associated 

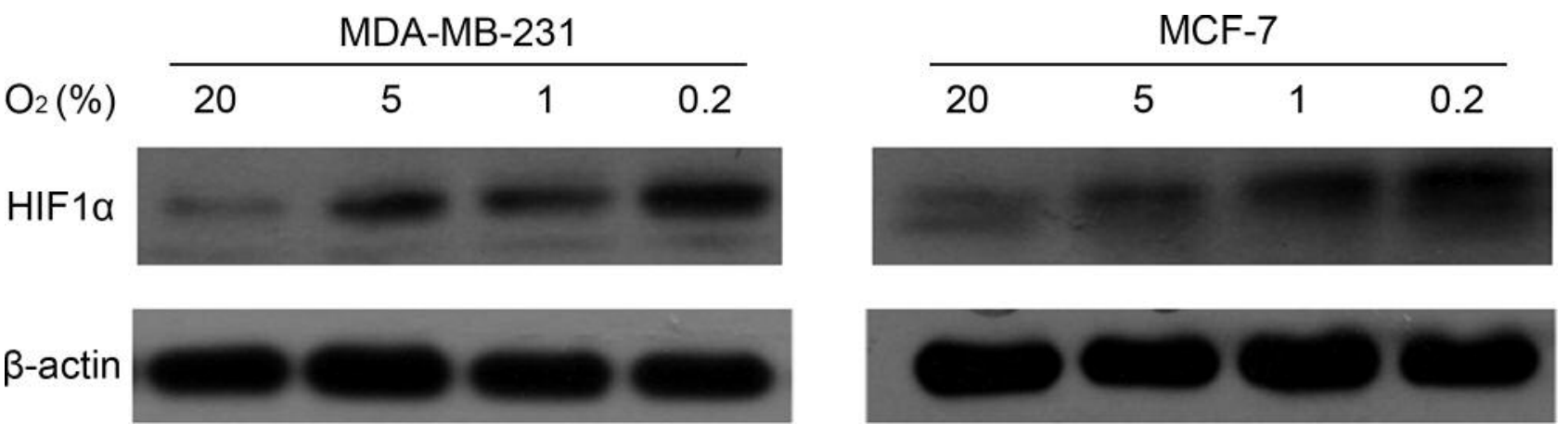

Figure 1. Protein levels of HIF1 $\alpha$ detected by western blot analysis in MDA-MB-231 and MCF-7 cells under various hypoxic environments.

circRNA and its expression level is associated with the progress of the tumor and may be a substitutive biomarker for HIF1 $\alpha$ in predicting the clinical impact of breast cancer.

\section{Materials and Methods}

Patients and tissue samples. Primary invasive ductal carcinomas of breast and adjacent non-cancerous breast tissues (referred to tissues more than $2 \mathrm{~cm}$ from tumors) were obtained from 30 female patients with breast cancer at the Breast Tumor Center, Sun Yat-Sen Memorial Hospital, Sun Yat-Sen University, from January 2016 to April 2017. Patients were divided into several groups based on age (13 cases $\geq 50$ years, 17 cases $<50$ years), tumor size ( 12 cases $\geq 5$ $\mathrm{cm}, 18$ cases $<5 \mathrm{~cm}$ ), lymph node (LN) status (16 cases with negative LN, 14 cases with positive $\mathrm{LN}$ ), estrogen receptor (ER) status (17 cases for ER-positive, 13 cases for ER-negative), human epidermal growth factor receptor 2 (HER2) status (12 cases for HER2-positive, 18 cases for HER2-negative).

Cell lines, experimental conditions and treatment. MCF-7 and MDA-MB-231 cell lines were purchased from American Type Culture Collection (Manassas, VA, USA) and cultured according to the recommended protocols. Cells were subjected to hypoxia by placing in a $\mathrm{CO}_{2}$ incubator and maintaining $20 \%, 10 \%, 1 \%, 0.2 \% \mathrm{O}_{2}$ by flushing nitrogen gas and $5 \% \mathrm{CO}_{2}$ at $37^{\circ} \mathrm{C}$ in automatic intelligent anaerobic culture system (MART II, Amsterdam, the Netherlands) for every $12 \mathrm{~h}$. For knock down the circDENND4C or HIF1 $\alpha$, specific siRNAs (Suzhou Jima Company, Suzhou City, China) were transfected with lipofectamine 3000 (Invitrogen, Carlsbad, CA, USA) into the cancer cells and $0.1 \mathrm{nmol}$ siRNA was used for each well of 6-well culture plates.

CCK-8 kit assay. Four groups of MDA-MB-231 cells were divided into mock (transfected with only lipo3000), nc (transfected with lipo3000 and nc siRNA), si-1 and si-2 (transfected with lipo3000 and specific siRNA1 or siRNA2). Cells were cultured in 6-well plates and digested by $0.25 \%$ trypsin (Introvigen) with siRNA transfection for 24 hours and added to 96-well plates with $100 \mu \mathrm{l}$ of 3,000 cells. CCK- 8 kits (Dojindo, Kumamoto, Japan) were used as inductions to evaluate the proliferation for four consecutive days and growth curves were drawn to show proliferation of cells dynamically. The first day's data were not detected because cancer cells were not attached to culture plates.

$R N A$ isolation and quantitative real-time polymerase chain reaction $(P C R)$. Total RNA was isolated using the TR RNA isolation kit (Invitrogen) and RNA was reversely transcribed into cDNA with a reverse transcription kit (Promega, Madison, WI, USA). CircDENND4C and HIFl $\alpha$ were detected by quantitative real-time PCR using the following primer sequences: CircDENND4C forward, 5'-CCCTTT GTCCTCTTTCAC-3' and reverse, 5'-ATAAGCCGTTCTTTCCCT-3', HIF $1 \alpha$ forward, 5'-GAACGTCGAAAAGAAAAGT CTCG-3' and reverse, 5'-CCTTATCAAGATG CGAACTCACA-3', $\beta$-actin forward, TCATGAAGTGTGACG TGGACATC and reverse, 5'-CAGGAGGA GCAATGATC TTGATCT-3'. $\beta$-actin was included as an internal control and the relative expression level of circDENND4C and HIFl $\alpha$ were normalized to $\beta$-actin. The data were analyzed using the $2^{-\Delta \Delta C t}$ method, where $\mathrm{Ct}$ represents the threshold cycle number which each amplified product was initially detected. This method, together with the $2^{-\Delta \Delta \mathrm{Ct}}$ method, was used to calculate the relative changes seen in gene expression from the real-time quantative PCR experiments.

Western blot analysis. Protein expression was detected by western blots as previously published (6). Antibodies against HIF1 $\alpha$ were purchased from Abcam (Cambrige, England, UK) (Concentration 5 $\mu \mathrm{g} / \mathrm{ml}$, Cat. \# GR194781-3) and antibody against $\beta$-actin was from Cell Signaling Technology (Danvers, MA, USA) (Concentration 1:1,000, Cat. \# 20536-1-AP).

Statistical analysis. All statistical analyses were carried out using SPSS 16.0 (New York, NY, USA). All in vitro experiments were performed independently for at least three repeats. Analysis of variance (ANOVA) analysis was used to evaluate the differences of 4 groups in proliferation assays each day. Student's $t$-test was used to evaluate the comparison of two independent groups in in vitro experiments or clinical subgroups. Linear regression analysis was used to analyze the correlation between expression of circDENND4C and HIF1 $\alpha$.

\section{Results}

HIF1 $\alpha$ expression was up-regulated in breast cancer cells under hypoxia induction. Since HIF1 $\alpha$ is the marker of the hypoxia process, we used western blotting to evaluate the 
A

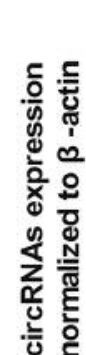

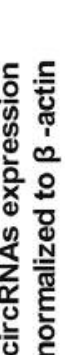
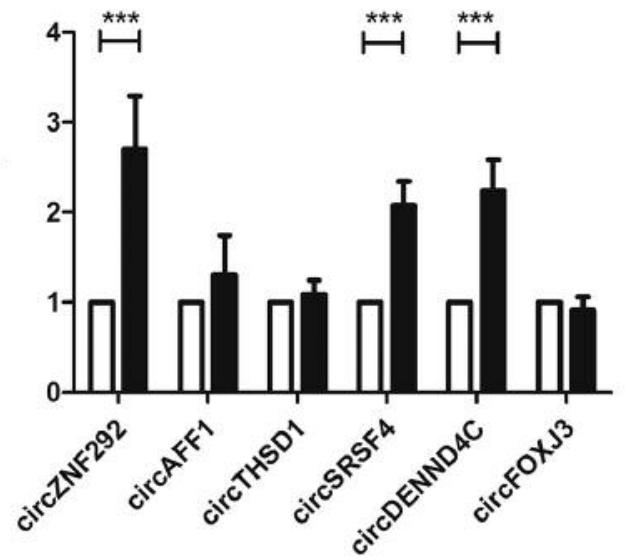

$$
\begin{aligned}
& * * *, P<0.001 \\
& 20 \% \mathrm{O} 2 \\
& 0.2 \% \mathrm{O} 2
\end{aligned}
$$

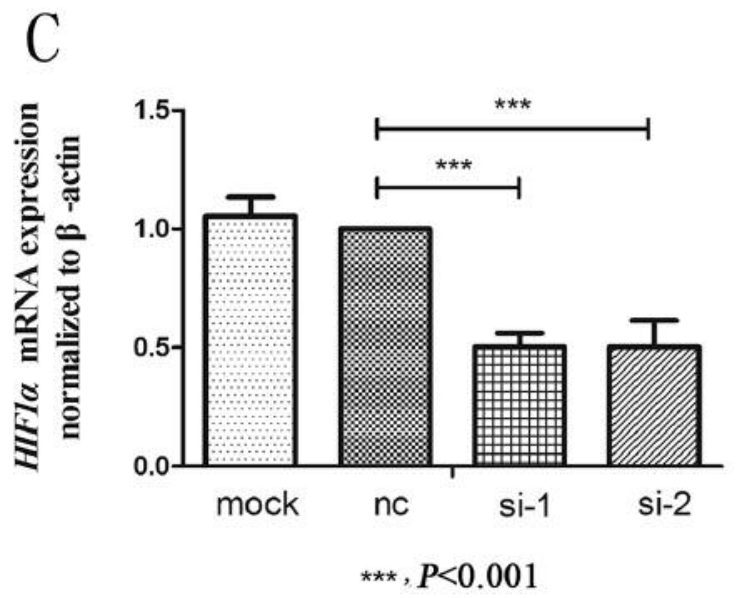

E

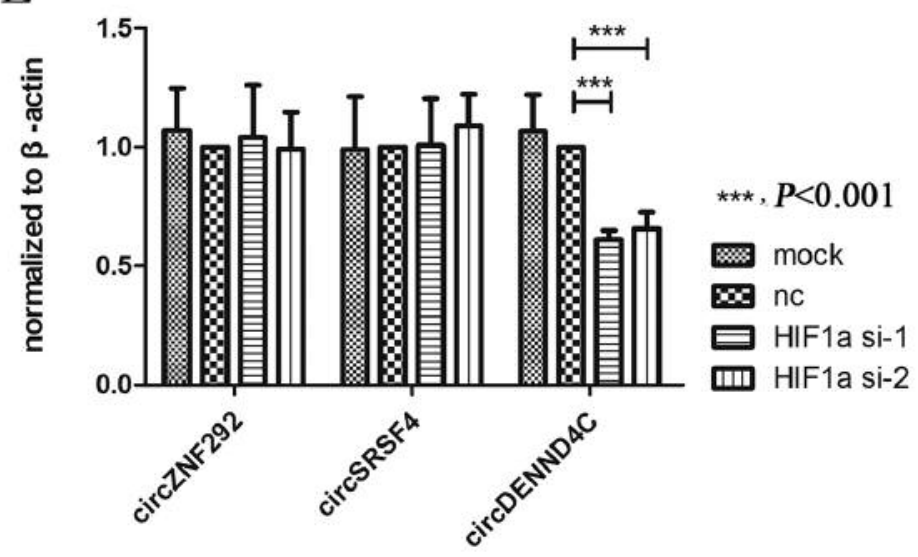

B MDA-MB-231
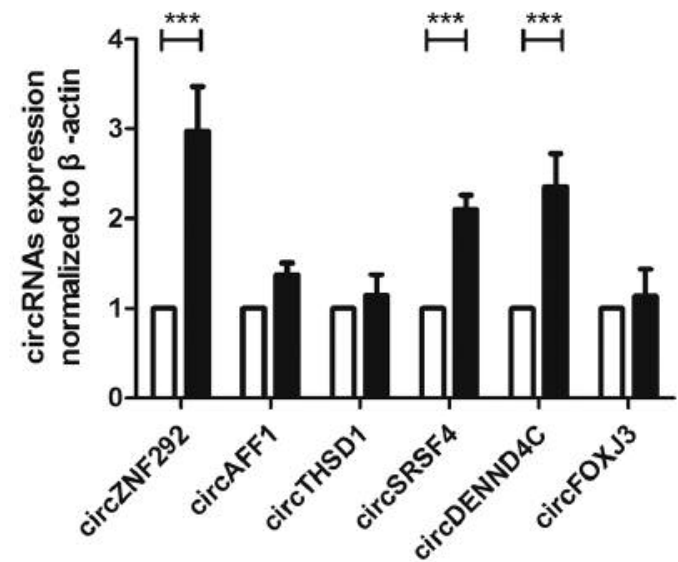

$$
\begin{aligned}
& * * *, P<0.001 \\
& \square 20 \% \mathrm{O}_{2} \\
& 0.2 \% \mathrm{O}_{2}
\end{aligned}
$$

D

mock nc si-1 si-2

\section{HIF1 $\alpha$}

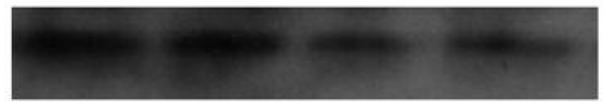

$\beta$-actin

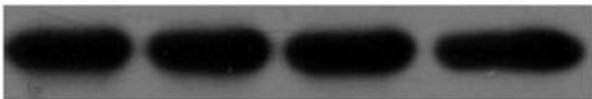

Figure 2. Hypoxia-associated or HIF1 $\alpha$-associated circRNAs selection by quantitative real-time polymerase chain reaction (qRT-PCR). A: Expression of candidate circRNAs in MCF-7cells in hypoxic and normoxic environments, *** $p<0.001$. B: Expression of candidate circRNAs in MDA-MB-231 cells in hypoxic and normoxic environments, ***p<0.001. C: HIF1 $\alpha$ mRNA level after knocked down by siRNAs, ***p<0.001. D: HIF1 $\alpha$ protein level after knocked-down by siRNAs. E: Expression of candidate circRNAs after silencing HIF1 $\alpha, * * * p<0.001$. 
expression changes of HIF1 $\alpha$ in MDA-MB-231 and MCF-7 cells under various oxygen concentrations $(20 \%, 5 \%, 1 \%, 0.2 \%$ $\mathrm{O}_{2}$ ) after 24-h treatments. The degree of HIF1 $\alpha$ in both MDAMB-231 and MCF-7 cells increased when the $\mathrm{O}_{2}$ concentration was reduced (Figure 1). The results showed that HIF1 $\alpha$ was a hypoxia-dependent protein in breast cancer cells with different molecular phenotypes. Because $0.2 \% \mathrm{O}_{2}$ was the most effective concentration for hypoxia induction, we carried out our following experiments under $0.2 \% \mathrm{O}_{2}$ concentration.

CircDENND4C is a HIFla-associated circRNA under hypoxia induction in breast cancer cells. From published literature we found that circRNAs circZNF292, circAFF1, circTHSD1, circDENND4C, circSRSF4 and circFOXJ3 were reported previously as hypoxia-associated circRNAs in endothelial cells after hypoxia induction (17). Here, to identify hypoxia-associated circRNAs, we detected these six circRNAs in MDA-MB-231 cells after hypoxia induction using qRT-PCR. We found that circZNF292, circSRSF4 and circDENND4C were up-regulated as oxygen concentration decreased, while the other three circRNAs did not display significant change (Figure 2A-B). To further detect whether circZNF292, circSRSF4 and circDENND4C were affected by HIF $1 \alpha$, we knocked down HIF $\alpha$ by specific siRNA in MDA-MB-231 cells in a hypoxic environment. Before subjecting the cells to hypoxia experiment, we confirmed that HIF1 $\alpha$-specific siRNAs successfully knocked down HIF1 $\alpha$ at both mRNA and protein levels in MDA-MB-231 cells after hypoxia induction (Figure 2C-D). Compared to negative control groups, only circDENND4C expression was decreased after silencing $H I F 1 \alpha$, while circZNF292 and circSRSF4 showed no obvious difference (Figure 2E). These results suggested that CircDENND4C was a HIF1 $\alpha$ associated circRNA after hypoxia induction.

CircDENND4C mediates proliferation of breast cancer cells in hypoxic environment. Since hypoxia is an essential factor that affects proliferation in cancer $(1,10-12)$ and expression of circDENND4C was increased after hypoxia induction or decreased after knocking down $H I F l \alpha$, we hypothesized that circDENND4C could mediate the proliferation of breast cancer cells. To explore the effect of circDENND4C on proliferation, we firstly used cell counting kit- 8 to detect proliferation viability of MDA-MB-231 cells after silencing circDENND4C by two specific siRNAs, which were confirmed to silence circDENND4C (Figure 3A). We found that circDENND4C did not affect proliferation of cancer cells in normoxic conditions (Figure 3B). We went on to subject the cells to a hypoxia condition in that the oxygen concentration was $0.2 \% \mathrm{O}_{2}$, as indicated earlier. We found that in this experimental setting, compared to the negative controls, silencing of either circDENND4C or HIFla resulted in decreased proliferation of cancer cells (Figure
3C-D). These results indicated that circDENND4C manifested its function in regulating proliferation only in HIF1 $\alpha$-dependent-hypoxia but not normoxic environments.

CircDENND4C expression level is positively correlated to HIF $1 \alpha$ level and tumor size in breast cancer patients. To further explore the clinical significance of circDENND4C in breast cancer patients, we firstly detected the expression level of circDENND4C in 30 paired cancer tissues and the adjacent normal tissues and found, by qRT-PCR, that tumor tissues highly expressed circDENND4C compared to the adjacent normal tissues (Figure 4A). Then, we detected the expressions of circDENND4C and HIFl $\alpha$ in 30 cases of breast cancer tissue, also by qRT-PCR, and analyzed their correlations. We found that the expression level of circDENND4C was positively correlated to HIF $1 \alpha$ mRNA level (Figure 4B). Since our functional assay showed that circDENND4C affected the proliferation of cancer cells in vitro, we next analyzed the relationship between circDENND4C and clinicopathological features of breast cancer patients (Table I). We found that circDENND4C level was associated with tumor size (Table I \& Figure 4C), while there was no significant correlation with the other clinicopathological features (Table I). All these clinical correlations were consistent with our finding in vitro and the already reported results that large tumors suffer more hypoxic damage than smaller ones (18). Our clinical analyses suggested that circDENND4C is a hypoxiaassociated circular RNA and has a clinical impact on breast cancer patients.

\section{Discussion}

In the present study, our in vitro assays revealed that circDENND4C expression was increased after hypoxia induction and decreased after silencing HIFl $\alpha$. Knockingdown circDENND4C inhibited proliferation of breast cancer cells in hypoxia environment. In clinical specimens of breast cancer, circDENND4C expression was more abundant in the tumor tissues than that of adjacent non-cancerous tissues and tumors with large size had increased circDENND4C expression level than those with small size. Our findings indicated that circDENND4C is a HIF1 $\alpha$-associated circRNA and its expression level is associated with the progression of tumor.

As increased cell proliferation of tumor promotes increased oxygen consumption (19-21), hypoxia is a feature of most solid tumors. To overcome hypoxic stress, cancer cells respond by increasing expression of $\operatorname{HIF} 1 \alpha$, a transcriptional factor in the nucleus responsible for transcription regulation of certain angiogenic and oncogenic factors. HIF $1 \alpha$ is unstable in a normoxic environment and rapidly degrades through the ubiquitin-proteasome pathway; 


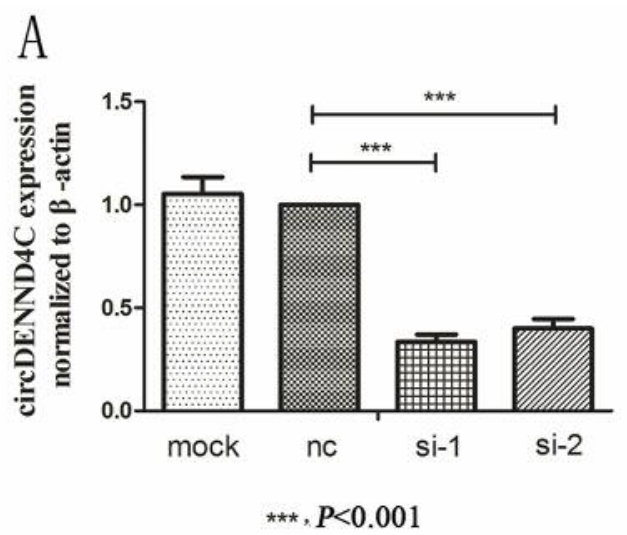

C

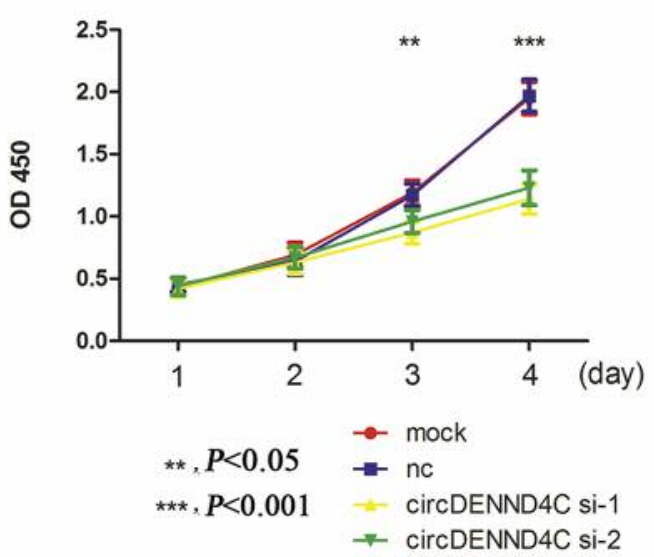

B

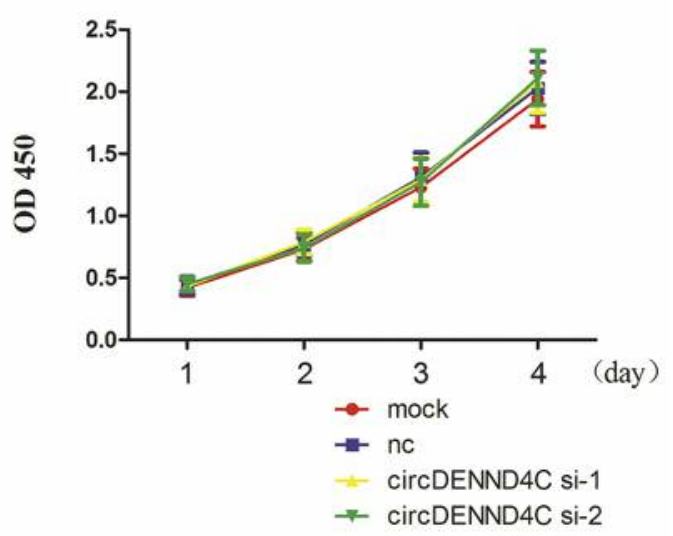

D

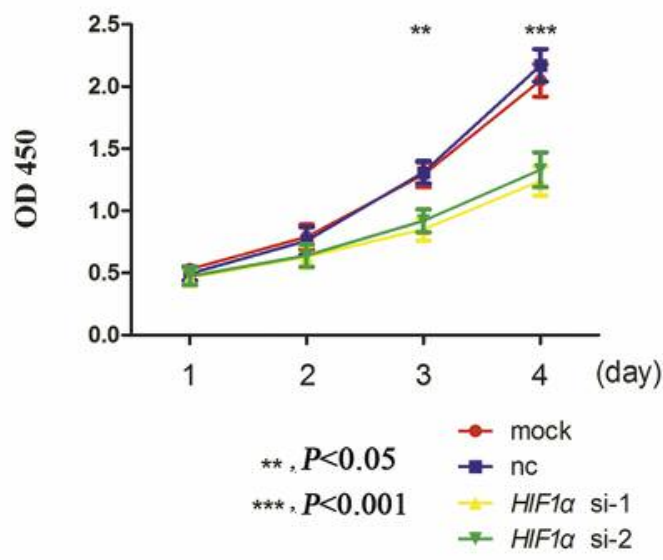

Figure 3. CircDENND4C affected proliferation of MDA-MB-231 under hypoxic conditions. A: CircDENND4C was silenced by specific siRNAs. B: Proliferation of MDA-MB-231 cells by interfering circDENND4C in nomoxic environment. C: Proliferation of MDA-MB-231 cells by interfering circDENND4C in hypoxic environment, $* * p<0.05, * * * p<0.001$. D. Proliferation of MDA-MB-231 cells by interfering HIF $\alpha$ in hypoxic environment, $* * p<0.05, * * * p<0.001$.

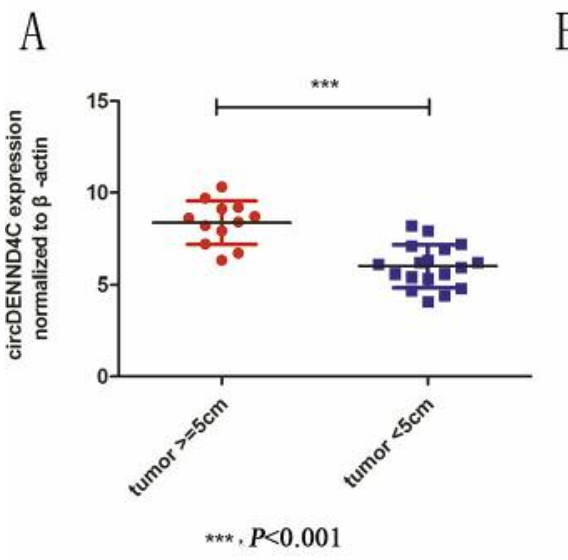

$\mathrm{B}$

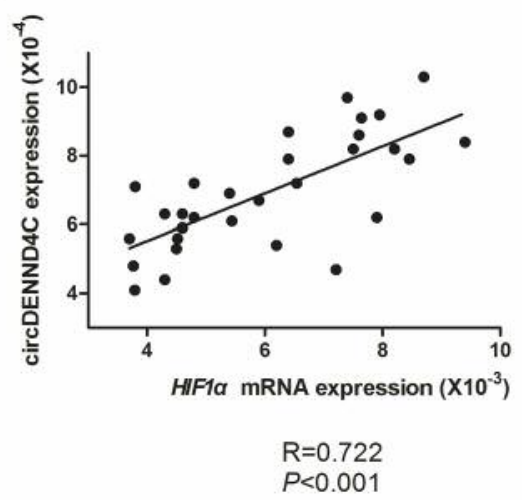

C

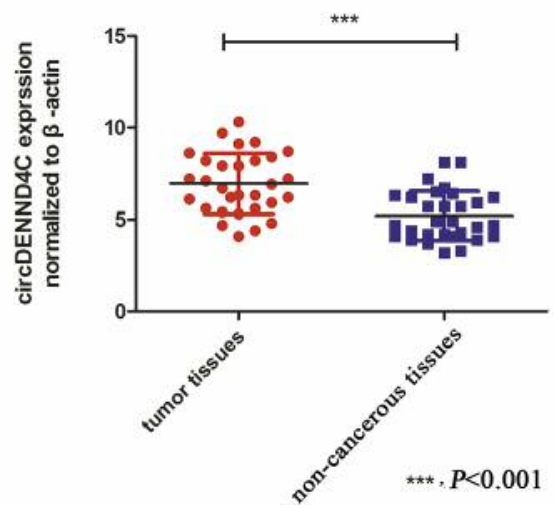

Figure 4. CircDENND4C expression detected in breast cancer tissues and correlation to HIF1a mRNA expression. A: CircDENND4C expressions in subgroup of tumor size, ${ }^{* *} p<0.001$. B: Correlation between mRNA levels of HIF1a and circDENND4C by linear regression analysis. $C$. CircDENND4C expression was detected by $q R T-P C R$ in tumors and matched adjacent non-cancerous breast tissues, $* * * p<0.001$. 
Table I. Correlation between clinicopathological features and circDENND4C expression in 30 cases of breast cancer tissues.

\begin{tabular}{|c|c|c|c|}
\hline \multirow[t]{2}{*}{ Parameter } & \multicolumn{3}{|c|}{ CircDENND4C expression } \\
\hline & $\mathrm{n}$ & Mean $\left(10^{-4}\right)^{*}$ & $p$-Value ${ }^{\#}$ \\
\hline \multicolumn{4}{|l|}{ Age } \\
\hline$>50$ years & 13 & 6.969 & \\
\hline$<50$ years & 17 & 6.918 & 0.9339 \\
\hline \multicolumn{4}{|l|}{ Tumor size } \\
\hline$>5 \mathrm{~cm}$ & 12 & 8.358 & \\
\hline$<5 \mathrm{~cm}$ & 18 & 5.994 & $<0.0001$ \\
\hline \multicolumn{4}{|l|}{ Lymph node\& } \\
\hline pNO & 16 & 6.971 & \\
\hline pN1-3 & 14 & 6.913 & 0.924 \\
\hline \multicolumn{4}{|l|}{ ER status $\$$} \\
\hline ER (+) & 17 & 7.189 & \\
\hline ER (-) & 13 & 6.509 & 0.283 \\
\hline \multicolumn{4}{|l|}{ HER2 status@ } \\
\hline HER2 (+) & 12 & 6.621 & \\
\hline $\operatorname{HER} 2(-)$ & 18 & 7.219 & 0.329 \\
\hline
\end{tabular}

*The mean of circDENND4C expression was estimated using the $2^{-\Delta \Delta \mathrm{Ct}}$ method or by equation, power $(2,-\Delta \mathrm{Ct}) .{ }^{\#}$ Student's $t$-test was used to analyze statistic differences in each subgroup. ${ }^{\&}$ Lymph node status: $\mathrm{pNO}$ indicates no lymph node metastasis and pN1-3 indicates at least one lymph node metastasis. ${ }^{\text {ER }}$ status shows estrogen receptor expression in breast cancer. ER (+) means that at least $10 \%$ of breast cancer cells express ER detected by immunohistochemistry (IHC), while ER (-) demonstrates a percentage less than $10 \%$. ${ }^{\circledR}$ HER2 status reveals expression of human epidermal growth factor receptor- 2 in breast cancer. HER2 (+) denotes strong (+++) HER2 expression by IHC and/or HER2 overexpression by fluorescence in situ hybridization (FISH). HER2 (-) represents a weak (- +) or moderate (++) HER2 level as reported by IHC and no HER2 overexpression by FISH.

however, a hypoxic environment can stabilize $\operatorname{HIF} 1 \alpha$, as HIF $1 \alpha$ is always detected for hypoxic level of tumor tissues. In this study, we confirmed that, in MDA-MB-231 and MCF-7 cells, the expression of HIF1 $\alpha$ was very low in a normoxic environment, but much more in an hypoxic environment, which was consistent with findings that HIF1 $\alpha$ is a transcriptional factor and helps cancer cells avoid hypoxic damage through regulating downstream genes, such as VEGF, MMPs, and some lncRNAs, such as GAPLINC (68). However, some literature focuses on the relationship between circRNAs and HIF1 $\alpha$-dependent hypoxia. We detected 6 potential hypoxia-associated circRNAs, including circZNF292, circAFF1, circTHSD1, circDENND4C, circSRSF4 and circFOXJ3, which were reported for the first time in endothelial cells (17), and found that circZNF292, circDENND4C, circSRSF4 increased after hypoxia treatment, while in all of these three circRNAs, only circDENND4C was decreased after knocking down HIFl $\alpha$ by specific siRNAs. These preliminarily data suggest that
circDENND4C was associated with HIF1 $\alpha$ in a hypoxic environment. Further functional assays in vitro confirmed that circDENND4C affected proliferation of cancer cells only in a hypoxic environment instead of in normoxic conditions demonstrating that HIF1 $\alpha$ might regulate the function of cancer cells via circDENND4C.

According to published articles, circRNAs are abundant and stable in cells and exhibit disease specificity, which made them ideal characteristics for diagnostic and therapeutic biomarkers $(13,22-24)$. In our study, compared to the matched non-cancerous tissue, primary breast cancer tumors have higher circDENND4C levels, indicating that circDENND4C might be a cancer-specific circRNA. As neovascularization occurs around edges of tumors, internal parts of larger breast cancer tissues always suffer from severe hypoxia; smaller tumors suffer slightly because the total tissues absorb enough oxygen and nutritional materials (1820). To some extent, the larger size of tumors sometimes means a faster proliferation. Therefore, tumor size can be an independent factor for hypoxia damage and results of proliferation. Indeed, we detected expression of circDENND4C in samples of human breast cancer and found that increased circDENND4C was associated with larger tumor size, rather than other indices, such as age, lymph node status, ER status and HER2 status. This clinical relevance suggests that circDENND4C may be a hypoxia-associated molecule having regulatory function in proliferation.

Hypoxia often occurs in the tumor environment, particularly in rapid growth of tumors and tumors of larger size. As a hallmark of hypoxia, HIF $1 \alpha$ has been a biomarker in predicting the clinical relevance in cancer; however, its predictive performance in clinics is limited, partly due to its instability in both normoxic and hypoxic environments. In contrast, circRNAs are abundant, stable and tissue-specific. Identifying HIF1 $\alpha$-associated circRNAs will provide more reliable and new biomarker candidates for clinical application. Our study discovered an HIF1 $\alpha$-associated circRNA and demonstrated its functional role and clinical relevance in breast cancer. These data provide evidence that circRNAs may be alternative biomarkers for predicting the clinical outcomes in cancer. However, there is still a long way to go in evaluating circRNAs' role as clinical predictors and the substitution of its parental genes.

\section{Acknowledgements}

This work was supported by grants from the National Key Research and Development Program of China (2017YFC1309100); the Natural Science Foundation of China (81472466 and 81672594); National Science Foundation of Guangdong Province (2014A03036003, 2014A030310378, 2014A020212059, 2015A030313172, 2015B050 501004, 2016A050502018 and 2016A030313237); China Scholarship Council (No. 201606385034); Cultivation for Major Projects and Emerging Interdisciplinary Funding Project of Sun Yat-sen University 
(17ykjc13) and Elite Young Scholars Program of Sun Yat-sen Memorial Hospital (Y201401). The Authors would also like to thank Cancer Research Wales and Wales National Life Science Research Network (NRN), Cardiff, Wales for their support.

\section{References}

1 Hubbi ME and Semenza GL: Regulation of cell proliferation by hypoxia-inducible factors. Am J Physiol Cell Physiol 309: C775C782, 2015.

2 Chang $\mathrm{J}$ and Erler J: Hypoxia-mediated metastasis. Adv Exp Med Biol 772: 55-81, 2014.

$3 \mathrm{Lu} \mathrm{X}$ and Kang Y: Hypoxia and hypoxia-inducible factors: Master regulators of metastasis. Clin Cancer Res 16: 5928-5935, 2010.

4 Zeng W, Liu P, Pan W, Singh SR and Wei Y: Hypoxia and hypoxia inducible factors in tumor metabolism. Cancer Lett 356: 263-267, 2015.

5 Wu H, Huang S and Zhang D: Autophagic responses to hypoxia and anticancer therapy in head and neck cancer. Pathol Res Pract 211: 101-108, 2015.

6 Pawlus MR, Wang L and Hu CJ: STAT3 and HIF1alpha cooperatively activate HIF1 target genes in MDA-MB-231 and RCC4 cells. Oncogene 33: 1670-1679, 2014.

7 Blick C, Ramachandran A, McCormick R, Wigfield S, Cranston D, Catto $\mathrm{J}$ and Harris AL: Identification of a hypoxia-regulated miRNA signature in bladder cancer and a role for miR-145 in hypoxia-dependent apoptosis. Br J Cancer 113: 634-644, 2015.

8 Liu L, Zhao X, Zou H, Bai R, Yang K and Tian Z: Hypoxia promotes gastric cancer malignancy partly through the HIF1 alpha dependent transcriptional activation of the long noncoding RNA GAPLINC. Frontiers in Physiol 7: 420, 2016.

9 Rundqvist H and Johnson RS: Tumour oxygenation: Implications for breast cancer prognosis. J Intern Med 274: 105-112, 2013.

10 Xie H, Ren X, Xin S, Lan X, Lu G, Lin Y, Yang S, Zeng Z, Liao W, Ding YQ and Liang L: Emerging roles of circRNA_001569 targeting miR-145 in the proliferation and invasion of colorectal cancer. Oncotarget 7: 26680-26691, 2016.

11 Hsiao KY, Lin YC, Gupta SK, Chang N, Yen L, Sun HS and Tsai SJ: Non-coding effects of circular RNA CCDC66 promote colon cancer growth and metastasis. Cancer Res 77(9): 2339-2350, 2017.

12 Zheng Q, Bao C, Guo W, Li S, Chen J, Chen B, Luo Y, Lyu D, Li Y, Shi G, Liang L, Gu J, He X and Huang S: Circular RNA profiling reveals an abundant circHIPK3 that regulates cell growth by sponging multiple miRNAs. Nat Commun 7: 11215, 2016.
13 Qu S, Yang X, Li X, Wang J, Gao Y, Shang R, Sun W, Dou K and $\mathrm{Li} \mathrm{H}$ : Circular RNA: A new star of noncoding RNAs. Cancer Lett 365: 141-148, 2015.

14 Li F, Zhang L, Li W, Deng J, Zheng J, An M, Lu J and Zhou Y: Circular RNA ITCH has inhibitory effect on ESCC by suppressing the Wnt/beta-catenin pathway. Oncotarget 6: 60016013, 2015 .

15 Li P, Chen S, Chen H, Mo X, Li T, Shao Y, Xiao B and Guo J: Using circular RNA as a novel type of biomarker in the screening of gastric cancer. Clin Chim Acta 444: 132-136, 2015.

16 Wang X, Zhang Y, Huang L, Zhang J, Pan F, Li B, Yan Y, Jia B, Liu $\mathrm{H}, \mathrm{Li} \mathrm{S}$ and Zheng $\mathrm{W}$ : Decreased expression of hsa_circ_001988 in colorectal cancer and its clinical significances. Int J Clin Expl Pathol 8: 16020-16025, 2015.

17 Boeckel JN, Jae N, Heumuller AW, Chen W, Boon RA, Stellos K, Zeiher AM, John D, Uchida S and Dimmeler S: Identification and characterization of hypoxia-regulated endothelial circular RNA. Circulation Res 117: 884-890, 2015.

18 De Jaeger K, Merlo F M, Kavanagh MC, Fyles AW, Hedley D and Hill RP: Heterogeneity of tumor oxygenation: Relationship to tumor necrosis, tumor size, and metastasis. Int J Radation Oncol Biol Phys 42: 717-721, 1998.

19 Denko NC, Fontana LA, Hudson KM, Sutphin PD, Raychaudhuri S, Altman $\mathrm{R}$ and Giaccia AJ: Investigating hypoxic tumor physiology through gene expression patterns. Oncogene 22: 5907-5914, 2003.

20 Hockel M and Vaupel P: Tumor hypoxia: Definitions and current clinical, biologic, and molecular aspects. J Nat Cancer Instit 93: 266-276, 2001

21 Padhani AR, Krohn KA, Lewis JS and Alber M: Imaging oxygenation of human tumours. Eur Radiol 17: 861-872, 2007.

22 Xuan L, Qu L, Zhou H, Wang P, Yu H, Wu T, Wang X, Li Q, Tian L, Liu M and Sun Y: Circular RNA: A novel biomarker for progressive laryngeal cancer. Am J Transl Res 8: 932-939, 2016.

23 Kulcheski FR, Christoff AP and Margis R: Circular RNAs are miRNA sponges and can be used as a new class of biomarker. $\mathrm{J}$ Biotechnol 238: 42-51, 2016.

24 Chen Y, Li C, Tan C and Liu X: Circular RNAs: A new frontier in the study of human diseases. J Med Genetics 53: 359-365, 2016.
Received May 2, 2017

Revised May 28, 2017

Accepted May 31, 2017 